\title{
A propósito de "De Caníbales”, de Michel de Montaigne: un pionero en el relativismo cultural
}

\section{"Of Cannibals", by Michel de Montaigne: a pioneer in cultural relativism}

\section{Sobre " Dos Canibais", de Michel de Montaigne: um pioneiro no relativismo cultural Cristo Manuel Marrero González ${ }^{1}$}

${ }^{1}$ Enfermero en el Servicio Canario de la salud. Área de salud de Tenerife (España). Doctor por la Universidad de La Laguna (España). Estudiante de Antropología Social y Cultural de la Universidad Nacional de Educación a Distancia (España). Orcid:0000-0002-6202-2411

Correspondencia: Calle Infante Juan Manuel 16, 38010. Santa Cruz de Tenerife. Tenerife. España. Correo electrónico de contacto:cmmarrerogonzalez@gmail.com

Cómo citar este artículo: Marrero-González, C. (2021). A propósito de “De Caníbales”, de Michel de Montaigne: un pionero en el relativismo cultural. Cultura de los Cuidados (Edición digital), 25(59). Recuperado de http://dx.doi.org/10.14198/cuid.2021.59.13

Recibido:11/11/2020 Aceptado: 18/01/2021

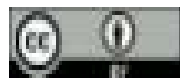

\section{RESUMEN}

En este documento se hace un análisis de uno de los ensayos, "De Caníbales", que escribió Michel de Montaigne (1533-1592) en la época renacentista. El objeto del presente documento es mostrar, mediante análisis reflexivo, las aportaciones que da este autor al llamado relativismo cultural desde el contexto histórico del siglo XVI. Dada la época, el ensayo es considerado "rompedor" dado el planteamiento y reflexión que hace el autor aproximándose a lo que más tarde en la Antropología se conocería como relativismo cultural en un tiempo donde la cultura europea se consideraba superior a la del resto de los pueblos. Al comparar las prácticas que hacen los precolombinos, relacionadas con el canibalismo, con las que hacen los europeos, el autor se da cuenta de que "nosotros" deberíamos hacernos mirar si nuestras prácticas realmente son mejores que las de los "otros"-los amerindios-. No obstante, hoy los planteamientos de Michel de Montaigne siguen estando presentes en un mundo donde se debate acerca de los 
Derechos Humanos y el relativismo cultural. Universalismo vs. Relativismo.

Palabras clave: Antropología; relativismo cultural; Derechos Humano; Michel de Montaigne; De Caníbales.

\section{ABSTRACT}

In this paper an analysis is made of one of the essays, "Of Cannibals", written by Michel de Montaigne (1533-1592) in the Renaissance period. The document's purpose is to describe, through reflective analysis, the contributions that this author gives to the so-called cultural relativism from the historical context of the 16th century. Given the time, the essay is considered "avant-garde" given the approach and reflection made by the author approaching what later in anthropology would be known as cultural relativism at a time when European culture was considered better than the rest of cultures in the world. By comparing the practices that preColumbians do, related to cannibalism, with those that Europeans do, the author realizes that "we" should think if our practices are really better than those of "others" -the Amerindians -. However, to this day, Michel de Montaigne's ideas are still present in a world where Human Rights and cultural relativism are debated. Universalism vs. Relativism.

Keywords: Antropology; cultural relativism; Human Rights; Michel de Montaigne; Of Cannibals.

\section{RESUMO}

Neste artigo, é feita uma análise de um dos ensaios, "Dos Canibalos", escrito por Michel de Montaigne (1533-1592) no período do Renascimento. O objetivo deste documento é mostrar, por meio de uma análise reflexiva, as contribuições que este autor dá ao chamado relativismo cultural a partir do contexto histórico do século XVI.

Dado o tempo, o ensaio é considerado "vanguardista", dada a abordagem e A reflexão feita pelo autor abordando o que, mais tarde, a antropologia seria conhecida como relativismo cultural, na época em que a cultura européia era considerada melhor do que o resto das culturas do mundo. Comparando as práticas que os précolombianos fazem, relacionadas ao canibalismo, com essas que os europeus fazem, o autor percebe que "nós" devemos pensar se nossas práticas são realmente melhores do que as dos "outros" - os ameríndios -. No entanto, até hoje, as idéias de Michel de Montaigne ainda estão presentes em um mundo onde os Direitos Humanos e O relativismo cultural é debatido. Universalismo vs. Relativismo.

Palavras-chave: Antropologia; relativismo cultural; Direitos Humanos; Michel de Montaigne, Dos Canibais.

\section{INTRODUCCIÓN}

Michel de Montaigne (1533-1592) escritor renacentista francés autor de una única obra titulada "Ensayos". En dicha obra, el autor, en uno de sus capítulos, se centrará en sus reflexiones acerca de la naturaleza de los indígenas de la América precolombina desmarcándose del pensamiento preponderante de su época. De Montaigne se acerca al llamado relativismo cultural para tratar a las gentes de estos pueblos.

En este documento se abordarán las ideas del autor en el capítulo "De Caníbales”, haciéndose una reflexión acerca del relativismo cultural, la idea del "buen salvaje” y del etnocentrismo imperante durante el sometimiento de América. Como señala Moreno Feliu (2014:396), la fuente en la que se basa Montaigne para hablar de estas etnias es Villegaignon quien emigró al actual 
Revista científica de la Asociación de Historia y Antropología de los Cuidados (Universidad de Alicante)

Brasil y le narraba sus vivencias años después de que se produjera el descubrimiento de América. Michel de Montaigne describe a esta persona como alguien sencillo que ha vivido de primera mano lo acontecido en esas tierras. Al ser una persona “simple”, posee un relato más veraz que el que pueda tener otra persona con mayor formación, y que en el empeño de argumentar pueden tener un discurso menos fiel a la realidad. Montaigne a lo largo de sus ensayos, observamos una narración de discurso llano, sencillo y cercano.

El autor ya nos deja entrever la importancia de no juzgar sin conocer, ni de llevarse por ideas preconcebidas. Alguien “culto”, podría estar condicionado para relatar de forma fiel lo que acontece en América debido a sus ideas preconcebidas. Como menciona Morales González (2018), en su estudio sobre Montaigne, una de las más bellas escuelas son los viajes que nos permiten librarnos del "violento prejuicio de la costumbre”, y, por ende, del prejuicio del etnocentrismo.

En toda la colección de Ensayos de este autor, hay un estilo característico por parte de este como filosofar sin filósofos, el uso de lenguaje llano y empleo de relatos extraordinarios. En "De caníbales”, sin lugar a duda, encontramos reflexiones argumentadas desde su punto de vista de persona en retiro y un lenguaje llano (Morales González, 2018).

La palabra “caníbal” deriva de “Caribe”. Colón había atribuido la palabra caníbal a las gentes que practicaban lo que desde la época griega se conocía como antropofagia (Moreno Feliu: 396).

\section{ACERCA DE “DE CANÍBALES”}

El autor comienza el capítulo haciendo ya una mención al concepto de bárbaro y a cuestionar el significado de tal concepto, construido desde el etnocentrismo. A ello se refiere al poner como ejemplo al rey romano Pirro que al tratar de avanzar con su ejército más allá de Italia apreció que a lo que los romanos llamaban bárbaros (pudiéndoles considerar inferiores que ellos), tenían una respetable estrategia militar. Así pues, ya Montaigne nos viene a anticipar que una cultura no tiene razón a ser superior a otras. En nuestro tiempo contemporáneo, occidente ha utilizado el término de “salvaje” de forma similar a cómo desde la Edad Antigua se utilizaba el de bárbaro (Levi-Strauss, 2012: 47-48). A partir de este punto, el autor reflexiona sobre si realmente son bárbaras las gentes de la América precolombina, según los testimonios de Villegaignon. ¿Por qué se les ha de considerar bárbaros por tener una comprensión de las cosas y de la naturaleza distinta de la cultura europea? Nuestra cultura no posee la verdad absoluta sobre todas las cosas. Además, Montaigne nos invita a pensar sobre la idea del buen salvaje de la que Jacques Rousseau hablaría más tarde durante la Ilustración (Moreno Feliu, 2014: 396). Los pueblos de la América precolonial obedecen a las leyes naturales. Ellos se rigen por la naturaleza, sin corromperse. Los hombres libres que se rigen por la naturaleza son nobles y no han sido adulterados con el pensamiento o cultura que se ha dado en el viejo continente. Tienen una mayor “inocencia”.

Montaigne al relatar sobre la geografía del lugar que Villegaignon le describe, encuentra similitudes con los antiguos relatos de la antigua Grecia cuando en estos se describen lugares más allá del estrecho de Gibraltar. Estos lugares eran de bosques frondosos, fértiles y extensos con grandes ríos. Se nos intenta transmitir que hay similitudes entre América y las descripciones de los lugares que la mitología griega nos narra. Sin embargo, pese a las similitudes que encuentra el autor, descarta que se trate de la Atlántida, por ejemplo. Hay un uso del modelo de la similitud, que se dio 
en el Renacimiento, para explicar la naturaleza de América (Moreno Feliu 2014: 157-159).

Raymond Williams (En Moreno Feliu, 2014: 221), al describir como palabra clave "naturaleza" nos habla de que precisamente la naturaleza del hombre-uno de los posibles sentidos que puede tener la palabra naturaleza-, se encuentra en los corazones bondadosos del ser humano alejados éstos del pensamiento artificial y corrompedor de los sujetos que viven en la Civitas. Montaigne coincide en mayor o menor medida con Joseph de Acosta al apreciar aspectos admirables en la cultura precolombina (Moreno Feliu, 2014:38-43). Estos grupos al vivir en el "estado de naturaleza”, como sociedades igualitarias, desconocen las más oscuras facetas del ser humano que hemos vivido desde que el hombre en nuestro contexto convive en sociedad $y$ no en comunidad: competitividad, maldad u odio. Montaigne nos expresa en que tal vez la tan perfecta República de Platón se quedaría lejos de la noble vida que mantienen los precolombinos.

He aquí pues que Montaigne, como argumenta Moreno Felíu (2014: 396), se desliga del pensamiento etnocentrista establecido en Europa y comienza a ser pionero en la argumentación del relativismo cultural al intentar transmitir que una cultura no es superior a otra, sino diferente y de que cada cultura se ha de comprender en sus propios términos. Solo se les puede llamar bárbaros porque tienen costumbres distintas, no porque sean inferiores 0 peores. Montaigne, se aleja del significado de bárbaro construido en la época en la que vivió. Las ideas de Montaigne entroncan con las ideas de Levi-Strauss en su manifiesto "Raza e Historia" para rechazar el etnocentrismo y centrarse en el relativismo cultural (Levi-Strauss, 2012: 47-53). LeviStrauss y Montaigne coinciden al rechazar la idea de bárbaro como alguien salvaje o no civilizado como se estaba utilizando desde la época griega, y aún en la renacentista.

“Cada cual llama barbarie a lo que no está acostumbrado cuando no se tiene otro ejemplo que el de uno. Peligrosa idea pensar que el mundo transcurre como sucede en casa.” (Morales González, 2018).

Nuestro ensayista nos describe la vida sencilla y "natural" en la que viven los indígenas en América, con sus costumbres y cultura. Se adentra en el "arte de la guerra" de estos pueblos. En esta parte Montaigne nos hace otra referencia al relativismo cultural al explicarnos qué hacen con sus prisioneros de guerra. Existe en estas culturas el canibalismo-recordemos el origen de la palabra caribe y canibalismo- al matar al prisionero y luego ser comido. Lejos de condenar esta práctica, o de horrorizarse ante la misma, se hace una reflexión intentando tener, creo, una "mirada distante” y se para a pensar que en nuestra cultura hacemos cosas tan horribles, o más, incluso, que el propio canibalismo como son las torturas que se hacían en la inquisición católica. De hecho, manifiesta que es más atroz quemar a una persona viva, previa tortura, que matarla y a posteriori devorarla.

Pero incluso, sin tener necesidad de llegar al canibalismo, los precolombinos no realizan torturas tan horrorosas como las inquisitorias. Se observa una apreciación a la idea de buen salvaje, incluso en esta faceta. Moreno Feliu (2014:86), nos explica que con la idea de bárbaro en Montaigne existe un doble sentido. Uno con la idea embrionaria del buen salvaje y el otro a nivel moral. ¿Quiénes somos nosotros para hablar de una moralidad superior, si hacemos cosas más atroces que las que hacen los propios indígenas supuestamente "no civilizados"? Los conquistadores del nuevo mundo utilizan un retrato de la condición humana del paradigma aristotélico consolidado en la Edad Media con las ideas cristianas e imperante en la época. Montaigne rompe con dicho paradigma (Raga Rosaleny, 2013). 
Revista científica de la Asociación de Historia y Antropología de los Cuidados (Universidad de Alicante)

Es entonces cuando volvemos a Levi-Strauss (2012: 49), cuando nos habla de la paradoja del relativismo cultural: “el bárbaro, en primer lugar, es el hombre que cree en la barbarie”. Referentes de la secta estoica como son Crisipo y Zenon pertenecientes a la cultura griega que ha inspirado a occidente y al Renacimiento en Europa, ya justificaban el canibalismo. Por tanto, cabe preguntarse, ¿quiénes son los bárbaros o salvajes? Cultura griega que para referirse a "los otros”, los llamaba bárbaros.

Michel de Montaigne no daba crédito al punto de vista según el cual el mundo se divide en una jerarquía natural de superiores e inferiores. Es así como está haciendo una crítica a la pedantería e imperialismo europeo que se daba invitando a hacer la reflexión de re-naturalizar al ser humano perdido cuya condición hemos perdido seamos caníbales, europeos, antiguos o modernos (Raga Rosaleny, 2013).

\section{CONCLUSIÓN Y REFLEXIÓN}

Ya en nuestra contemporaneidad, el relativismo cultural sigue encontrándose en un profundo debate con personas a favor $\mathrm{y}$ detractores. Existe un conflicto entre relativismo y universalismo, es decir, relativismo cultural y Derechos Humanos. La reflexión de Hinton que recoge Moreno Feliu (2014:88), nos hace pensar de las dificultades que se presentan para responder o condenar hechos que pueden ocurrir en determinadas culturas como son los genocidios, dado que se puede incurrir en una actitud etnocéntrica.

De todas formas, antes de realizar cualquier juicio de valor sin conocimiento de causa, habría que adentrarse en una cultura determinada para poder hacer realmente una valoración y hacer un examen de reflexión sobre nuestra cultura y la de "los otros": quizá hacer uso de la "mirada distante". Montaigne en el siglo XVI lo hizo, como hemos visto. Incluso, tal vez, hizo uso también de la mirada distante. Kottak (2011:41-42), nos transmite que con el relativismo cultural no podemos usar ideas estandarizadas de una sociedad determinada para evaluar una conducta que se da en otra cultura. En paralelo a los derechos humanos, Kottak (2011:42) habla de que existen los Derechos Culturales. Cosa que quizás Herskovits echó en falta al elaborarse la carta de los Derechos Humanos por parte de Naciones Unidas (Moreno Feliu, 2011:86; 376-381). La inspiración de la misma parte del siglo XVIII, en la que se dio la Ilustración y la Declaración de los Derechos del Hombre y del Ciudadano en Francia. Es decir, existe una concepción occidental al haberla elaborado sin tener en cuenta al resto de culturas. Además de que se ha obviado que los seres humanos convivimos en grupos culturales, y la cultura es la que nos construye como tales. La inspiración de la que parte la elaboración de los Derechos Humanos se ha quedado obsoleta teniendo en cuenta el etnocentrismo presente de la época.

Al volver a Kottak (2011:43), éste intenta transmitir la importancia de que por encima del relativismo cultural existen unos estándares universales de justicia y moralidad que no deben ser obviados. Las antropólogas no deben aprobar costumbres que estén relacionadas con el infanticidio, el canibalismo o la tortura. Así pues, Kottak refiere que hay antropólogos que se niegan realizar trabajo de campo en determinados lugares porque les parecen horribles ciertas prácticas que se dan. El debate es arduo y complejo. Tal es así que Kottak termina el párrafo y le hace la siguiente pregunta al lector: “¿Qué opina usted?” (Kottak, 2011:43). Desgraciadamente, los antropólogos durante los siglos XIX y XX para mantener una "objetividad científica" del relativismo cultural hacían la vista gorda para estudiar a las culturas, aunque se dieran hechos tan atroces como son las masacres o 
torturas humanas. Parece entonces un gran logro para la Antropología haber reflexionado sobre esta actitud que se daba durante el modelo clásico (Moreno Feliu: 87-88). Sin Democracia, sin autogobierno donde los ciudadanos puedan ejercer derechos y tener libertades, no puede haber un Humanismo. La Antropología como disciplina no puede ser "una mera espectadora” y neutral sobre lo que acontece en un lugar y tener espíritu crítico (GarcíaAlonso, 2016: 14-17).

No obstante, los Derechos Humanos no son entes estáticos, y como se promulgó en la Asamblea de la Asociación Americana de Antropología en 1999 (En Moreno Feliu, 2014: 392-393), la comprensión de los Derechos Humanos evoluciona constantemente y la Antropología debe contribuir al conocimiento activo de la condición humana. La Antropología debe ser protagonista en lo que al relativismo cultural se refiere. Del respeto por los derechos culturales y de encontrar un acuerdo moral universal para determinar aquello que es susceptible de ser condenado en cualquier cultura, incluida la nuestra. Algo que puede ser condenable en nuestra cultura europea, es el hecho de cómo se ha dado la acogida a los refugiados sirios en estos últimos años, quedándose Europa "muy corta” a la hora de darles asilo político. El asilo político es un Derecho Universal Humano.

Tal y como defiende Benhabib (2008), existe un derecho moral fundamental, el "derecho a tener derechos" de todo ser humano, esto es, de ser reconocido por otros y de reconocer a otros a su vez como persona merecedora de respeto moral y de derechos legalmente garantizados en el seno de una comunidad humana. Reflexiono y pienso que nos comportamos como bárbaros por como tratamos al otro: en sociedades o países donde la inmigración ha aumentado, las ideas u opiniones de origen etnocéntrico han terminado por crear corrientes de opinión al ver al inmigrante como un problema xenofobia- (Alaminos et al, 2010).

Pese a que observamos a Montaigne como un vanguardista en su pensamiento, el economista Amartya Sen argumenta que por ejemplo la defensa de la tolerancia universal se promueve en la India muchos siglos antes de que se promoviera en Occidente, como se recoge en el ensayo de García-Alonso (2016:20). Este hecho es relevante para darnos cuenta de que Occidente no tiene "verdades absolutas" o superiores. La autocrítica que hemos de hacer en nuestra civilización occidental mirando no solo al presente, sino a nuestra historia reciente en el siglo XX, debe ser profunda y saber aprender de los que llamamos “otros”. El trato que damos a las comunidades minoritarias en diferentes países no es de recibo.

Aún como estudiante de Antropología Social y Cultural, me queda mucho por observar, reflexionar, leer y comprender. Admiro a Montaigne porque ya en su época desafía al pensamiento establecido. Parece que su tiempo de reflexión le hizo ser un adelantado a su tiempo. Es “chocante” como en dicha época se pudiera cuestionar sus propias prácticas, no considerándolas mejores que las de los nativos americanos. Ha sido un cuestionamiento al pensamiento autoritario de la época, al examinar a su propia sociedad y detenerse en que también nosotros podemos ser horribles o más que las culturas amerindias. Por ello, traslado a usar sus reflexiones a este siglo XXI y reflexionar sobre si aún nosotros hacemos prácticas 0 tenemos costumbres que pueden ser condenables antes de juzgar a las de otras culturas. Como hicieron Montaigne o LeviStrauss. Porque para raros, nosotros (Bohannan, 1996).

Pero hago una reiteración a la argumentación que anteriormente realizaba Kottak (2011: 43), en que, si en una cultura 
Revista científica de la Asociación de Historia y Antropología de los Cuidados (Universidad de Alicante)

se dan casos como los infanticidios, por ejemplo, existan cláusulas morales universales para en determinados casos condenar algo propio de una cultura. La Antropología podría instar a reelaborar una carta de Derechos Humanos donde se tengan en cuenta los puntos de vista de los demás pueblos del mundo, incluidos los pueblos indígenas. Es necesario concebir al ser humano no como un ente individual único, sino que se forja gracias a un grupo cultural. Y además sería necesario incluir dichas cláusulas morales universales. Es muy ambicioso, tal vez quijotesco, pensar así, pero, la Ilustración se dio en un momento histórico determinado con un pensamiento concreto y nuestra época es muy distinta a la de aquel de entonces. De forma prudente, puedo decir que Montaigne estuviera más adelantado, en parte, a ilustrados como Rousseau que nombraba la idea del "buen salvaje” también recurrente en la escuela literaria del Arcadismo (Cándido, 1968: 1520). Así, Rousseau al imaginar las características del lenguaje primitivo (Velasco Maillo, 2003: 29-41), apreciaba a los pueblos ágrafos como salvajes al no considerar que éstos tuvieran una lengua como tal. Los pueblos ágrafos, como los indios, serían reductos de hombres primitivos o no suficientemente evolucionados para la época del siglo de las luces.

No quisiera pasar por alto la posible sabiduría que nos brinda Michel de Montaigne a Enfermería. Quizá la "moraleja” que podamos aprender esté relacionada con las personas de otras culturas a las que brindamos atención y cuidados: competencia cultural (Spector, 2016). En muchas ocasiones presentamos muchos prejuicios e ideas preconcebidas al atender a una persona migrante $\mathrm{y}$ comprendemos que, al practicar otras costumbres, que pueden "chocarnos", tenemos juicios etnocentristas. Hace algunos años, ya reflexioné acerca de la importancia de la competencia cultural (Marrero, 2013) y el deseo de querer conocer al otro para dar una mejor atención.

Dar formación sobre Competencia Cultural en las profesiones sanitarias no deja de suponer un reto porque a nivel perceptivo-cognitivo es muy difícil desprenderse del etnocentrismo. Se necesita hacer mucha autocrítica de nuestros valores y creencias antes de criticar a los del resto como bien de forma tan llana y sencilla nos invitaba Montaigne. Desde luego, desde la Academia o Universidad se debe de fomentar el uso del juicio en Enfermería en las asignaturas relacionadas con Teorías y Métodos en Enfermería o en Ética. El juicio, o razonamiento crítico, y de actuación de una enfermera ante el cuidado de personas de distintas culturas no puede partir con etnocentrismo sino con sensibilidad cultural y ponerse en el lugar del que llamamos "otro" -el otro no está fuera de la cultura, sino que es constitutivo de ella (Benhabib, 2008)-. Quizá eso nos aconsejara Michel de Montaigne, porque en este mundo cada vez más globalizado y a la vez más desigual, habrá que tener sensibilidad cultural y tampoco caer en lo que la filósofa Adela Cortina (2017), ha acuñado como aporofobia.

\section{BIBLIOGRAFÍA}

Alaminos, A., López, C., \& Santacreu, O. (2010). Etnocentrismo, Xenofobia y Migraciones Internacionales en una Perspectiva Comparada. Convergencia, Revista de Ciencias Sociales, 17(53), 91-124.

Bohannan, P. (1996). Para Raros, Nosotros. ( $2^{\mathrm{a}}$ reimpresión) Madrid: Editorial Akal.

Benhabib, S. (2008). Otro universalismo: Sobre la Unidad y Diversidad de los Derechos Humanos. Isegoría, (39), 175-

203. 
Cultura de los Cuidados

https://doi.org/10.3989/isegoria.2008.i39.62 7

Cándido, A. (1968). Introducción a la Literatura de Brasil. Madrid:Monte Ávila Editores.

Cortina, A. (2017). Aporofobia, el Rechazo al Pobre: un Desafio para la Democracia. Barcelona: Paidós.

Feliu, P. M. (2014). De lo Lejano a lo Próximo: un Viaje por la Antropología y sus Encrucijadas. Madrid:Editorial Universitaria Ramón Areces.

García-Alonso, M. (2016). Ética y Antropología. ( $2^{\mathrm{a}}$ reimpresión) Madrid: Editorial UNED-Universidad Nacional de Educación a Distancia-.

Kottak, C.P. (2011). Antropología Cultural. (14 ${ }^{\text {a }}$ edición). México DF: McGraw Hill. ISBN:978-607-15-0555-2.

Lévi-Strauss, C. (2012). Raza y cultura. (5 edición). Editorial Cátedra. ISBN: 978-84-376-1188-4.

Marrero González, C. M. (2013). Competencia Cultural. Enfoque del Modelo de Purnell y Campinha-Bacote en la Práctica de los Profesionales Sanitarios. ENE, Revista de Enfermería, 7(2).

Morales González, J. (2018). Estudio y Sabiduría Popular en los Ensayos de Michel de Montaigne. En Claves del Pensamiento. Revista de Filosofia, Arte, Literatura, Historia, 12(24), 57-83.

Raga Rosaleny, V. (2013). Cultura y Naturaleza: Montaigne en América. Alpha (Osorno), (37), 91-104. Disponible en: http://dx.doi.org/10.4067/

S0718-22012013000200007

Spector, R.E. (2016). Cultural Competence. Cultura de los Cuidados, 44(20), 9-14.Recuperado de

http://dx.doi.org/10.14198/cuid.2016.44.01

Velasco Maillo, H. (2003). Hablar y Pensar, Tareas Culturales. Temas de Antropología
Lingüística y Antropología Cognitiva.

(6 ${ }^{\mathrm{a}}$ reimpresión). Madrid: Editorial UNED.

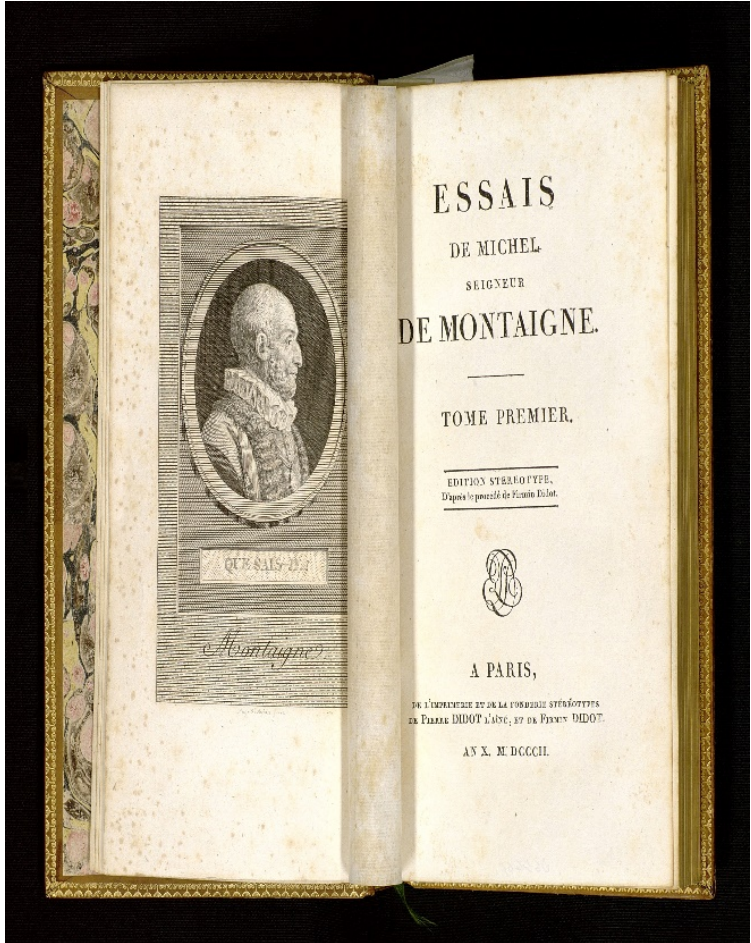

